\title{
TWO CASES OF UNILATERAL PARALYSIS OF THE DIAPHRAGM IN THE NEWBORN TREATED SURGICALLY
}

\author{
BY \\ J. A. W. BINGHAM \\ From the Royal Belfast Hospital for Sick Children
}

(RECEIVED FOR PUBLICATION FEBRUARY 3, 1954)

Unilateral paralysis of the diaphragm in the newborn infant is not a common condition. It does not appear to be well known in this country, for there have been no reports of the condition in the British literature. It is, however, a serious condition, and is often fatal because of respiratory difficulty and, to a lesser extent, of the feeding difficulties always associated with dyspnoea in infants. This contrasts markedly with the absence of any such respiratory difficulty from diaphragmatic paralysis in adult life or later in childhood and is due to the very mobile mediastinum of the infant becoming markedly displaced, so that, in addition to the lung on the side of the paralysed diaphragm being compressed, the lung on the opposite side also becomes compressed. This is clearly seen in Figs. 1A and 4A.

The condition has been reported most often on the right side. It may be associated with a brachial plexus injury of the Erb type or it may be an isolated lesion. Schifrin, writing in 1952, was able to collect from the literature nine cases of isolated paralysis of the diaphragm in the newborn, all

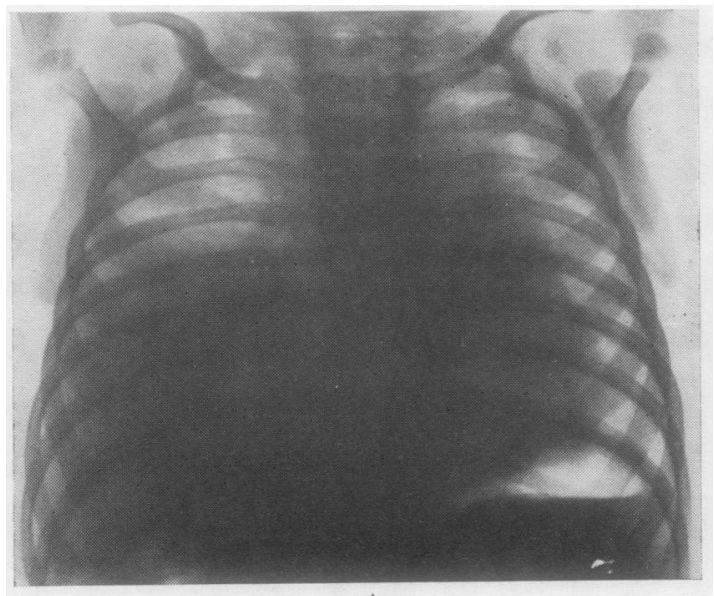

from American and German sources, and he added two further cases. The following two cases of isolated diaphragmatic paralysis are, therefore, the twelfth and thirteenth to be reported. Incidentally, none of the previously reported cases have been treated surgically, nor, even when ending fatally, does operation appear to have been considered.

\section{CASE RePorts}

CASE 1.-Yvonne B., aged 12 weeks, was on June 16, 1951, admitted to the Royal Belfast Hospital for Sick Children under the care of Dr. Muriel Frazer, who later kindly referred the case to me. The infant had been delivered as a breech presentation with extended limbs, and had been so cyanosed and dys-

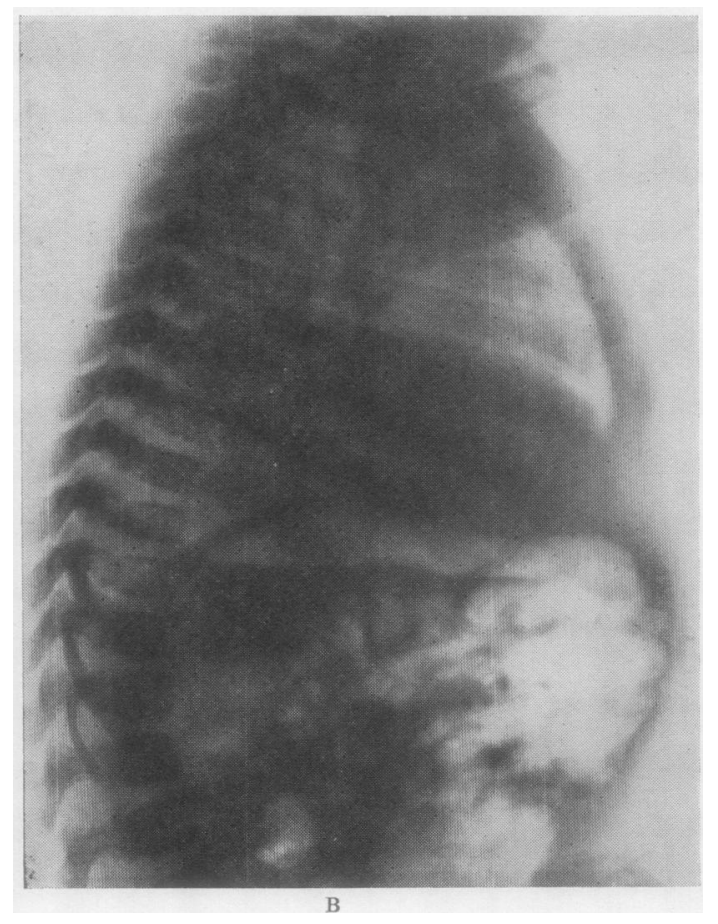

FIG. 1.-Case 1: pre-operative radiographs. In $A$ the lower half of the right chest is opaque and the mediastinum shifted to the left. $B$ is a lateral view showing clearly the raised leaf of diaphragm. 
pnoeic after birth that she had been kept in an oxygen tent for three days. Afterwards she had had repeated attacks of cyanosis and dyspnoea, and had failed to thrive properly. During some weeks there had been a weight gain and during others a weight loss. In the 12 weeks since birth her weight had only increased from $8 \mathrm{lb}$. to $9 \mathrm{lb} .8 \mathrm{oz}$. Five other children in the family were alive and well, the only serious illness having been rheumatic fever in one child.

On examination, the infant was seen to be dyspnoeic, slightly cyanosed, and undernourished. There was little else abnormal to be found, the most striking finding being recession of the abdomen on inspiration. There was also relative dullness and weak air entry at the right base.

A radiograph of the chest showed a marked shift of the heart and mediastinum to the left, and the lower half of the right chest was seen to be opaque (Fig. 1A). It was not clear from this film what the condition was, but a right lateral radiograph made it clear that the right dome of the diaphragm was very high (Fig. 1B), and that therefore the opacity occupying the lower half of the right chest on the postero-anterior radiograph was due to the liver. Subsequent screen examination showed paradoxical movement of the raised right side of the diaphragm.

During the following week dyspnoea and attacks of cyanosis continued and the infant lost $10 \mathrm{oz}$. It was felt that because of her continued deterioration an attempt should be made to correct the condition by operation.

At operation (June 28, 1951) under endotracheal nitrous oxide, ether, and oxygen anaesthesia (Dr. Maurice Brown) a right thoracotomy was performed through the bed of the ninth rib. The diaphragm was seen to extend high into the chest. It was not thin and attenuated, but a musculo-aponeurotic sheet of normal appearance and thickness. An incision was made across the diaphragm about 3 or $4 \mathrm{~cm}$. from its costal attachment. The two portions were then overlapped, the peripheral portion being fixed by a series of interrupted stitches underneath the central portion so that the cut edge of the central portion could then be secured by stitches round the costal attachment of the diaphragm. The chest was closed without drainage.

The result of the operation exceeded anything that could have been expected. There were no further attacks of dyspnoea or cyanosis, and within 24 hours the infant was taking her feeds well and putting on weight. Indeed, between the fifth and ninth postoperative day, when the child was discharged home. her weight increased by $8 \mathrm{oz}$, a third of the total weight gain during the 12 weeks before admission.

Post-operative radiographic examination showed that, though the right side of the diaphragm was at a considerably lower level than before, it was still raised and was still moving paradoxically. However, the heart and mediastinum had returned to their normal positions and there was much more aerated lung on both sides (Fig. 2).
The parents failed to bring the child for a followup examination and she was not seen again until two years later. She was then a fine, sturdy, healthy-

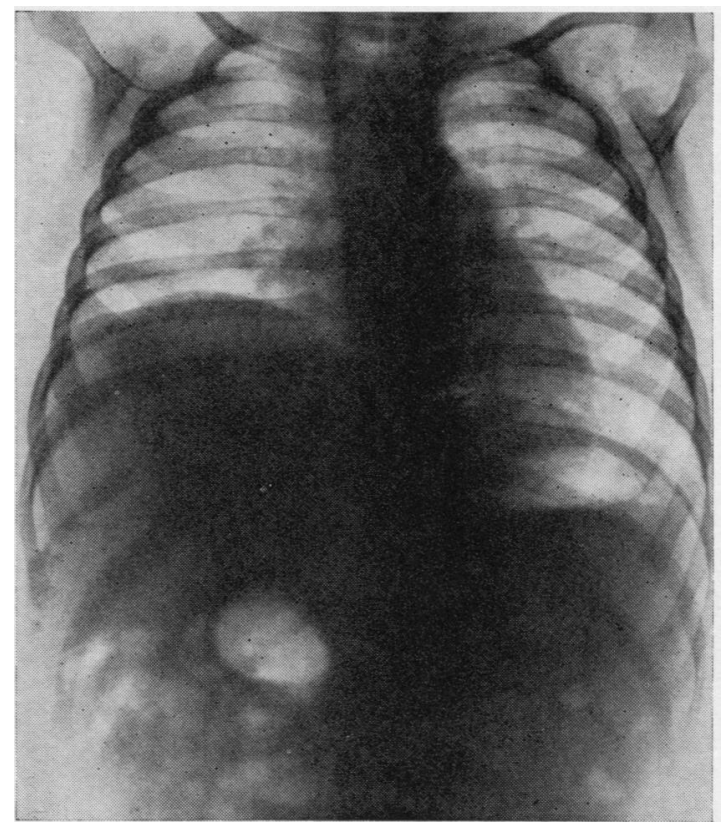

FIG. 2.-Case 1: radiograph a few days after operation showing the decreased elevation of the diaphragm, the return of the miediastinum towards the mid-line, and the increased aeration of both lungs.

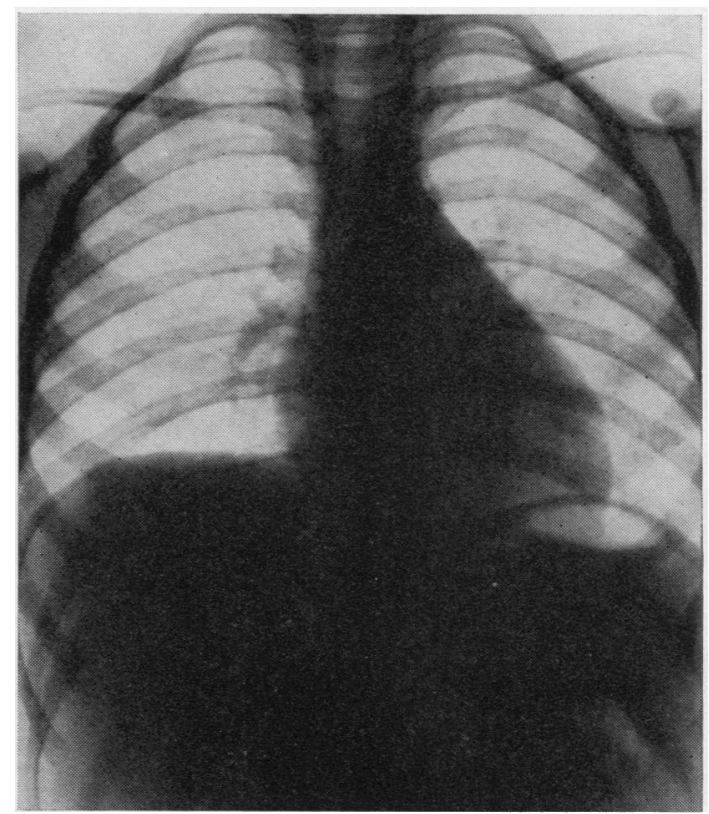

FIG. 3.-Case 1: radiograph two years after operation showing the completely normal appearance. 
looking little girl. A radiograph of the chest (Fig. 3) showed a completely normal appearance, the right side of the diaphragm being at a normal level, and on screening it was seen to be moving synchronously on respiration.

CASE 2.-Marie O'R. had also been a breech presentation with extended arms and legs. After birth, which was at Downe Hospital on May 11, 1953, she had been very cyanosed and difficult to resuscitate. A greyish cyanosis remained and rapid breathing was constant. Attempts at feeding were usually associated with increased cyanosis and dyspnoea, only small feeds be.ng taken and these slowly. A radiograph of the chest was interpreted as showing collapse of the right lower and middle lobes and partial collapse of the left lung. When 10 days old the child was transferred to the Royal Belfast Hospital for Sick Children. Here I was asked to see her by Dr. A. A. H. Gailey.

Further radiographs were taken and the lateral view again made the diagnosis clear by showing the smooth curve of the right dome of the diaphragm extending high across the chest (Fig. 4). Clinical findings were similar to those in the previous case, there being dullness and poor air entry at the right base. The infant had repeated attacks of increased cyanosis, especially on feeding. These attacks were at times alarming, and on one occasion, 28 days after admission, the child appeared to be virtually dead after a cyanotic attack but with vigorous resuscitative measures she revived. I operated on her a few days later. Her birth weight of $7 \mathrm{lb}$. had fallen to $6 \mathrm{lb} .11 \mathrm{oz}$.

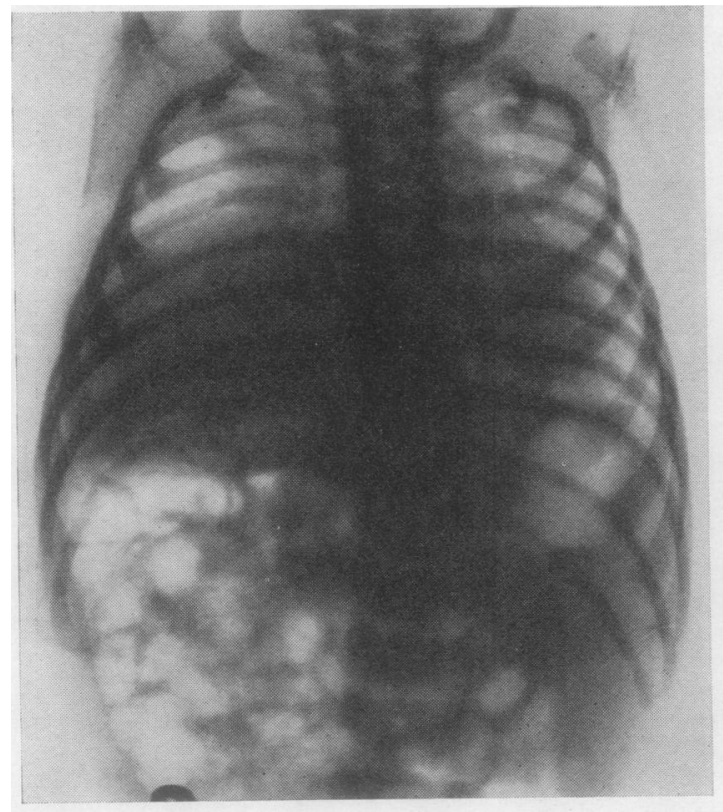

At operation (June 24,1953 ) the anaesthetic technique was as in the first case (Dr. S. H. Love). Thoracotomy was performed through an intercostal incision over the lower part of the right chest. As in the previous case, the appearance of the diaphragm was normal, except that it extended almost to the apex of the chest. Incision across the diaphragm and overlapping of the two portions was again carried out, but on this occasion a portion of the diaphragm was excised and sent for histological examination. The chest was closed without drainage.

The pathologist's report on the portion of diaphragm excised was as follows (Dr. J. M. Drennan) :

"The muscle fibres are swollen and hyalinized and have lost their pattern of striations. There seems to be much atrophy of the fibres and increased cellularity due to nuclear proliferation. The general picture of muscle degeneration is consistent with a nerve lesion and does not in any case suggest a primary muscle lesion. The tissue pattern is of normal type : there is nothing to indicate a defect of development (Fig. 5)."

Within a day or two of operation the child was taking her feeds well and putting on weight steadily. Dyspnoea and cyanosis were completely relieved. When discharged home 22 days after operation her weight had increased by $17 \mathrm{oz}$.

A post-operative radiograph showed the right dome of the diaphragm to be lower in the chest, the heart and mediastinum to have returned towards the midline, and both lungs to be much better aerated (Fig. 6).

Five months after operation, the infant looked fit $\overrightarrow{\bar{o}}$ and well and extremely well nourished. There had

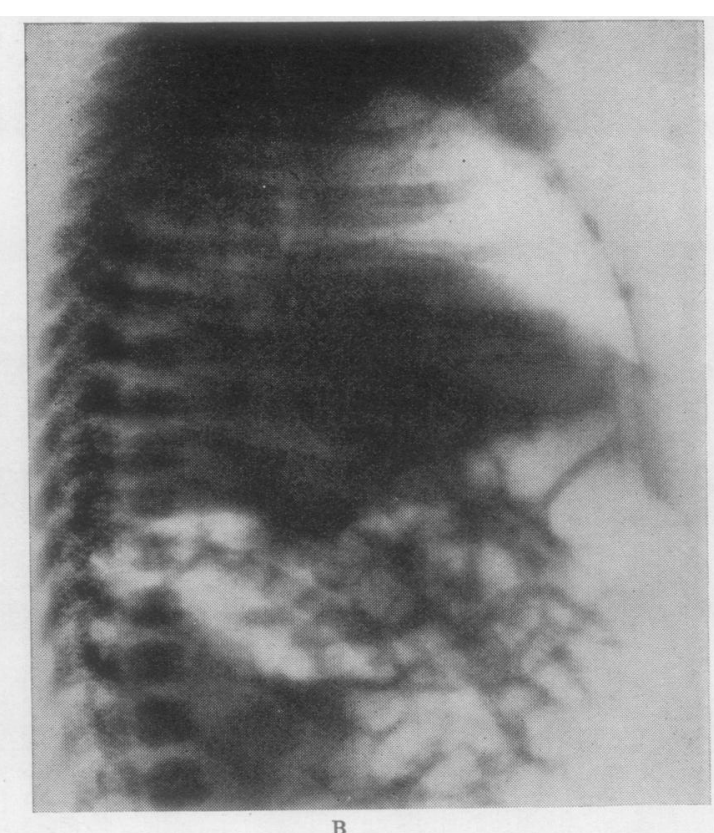

FIG. 4.-Case 2: A and B are pre-operative radiographs, strikingly similar to those in Case 1. 


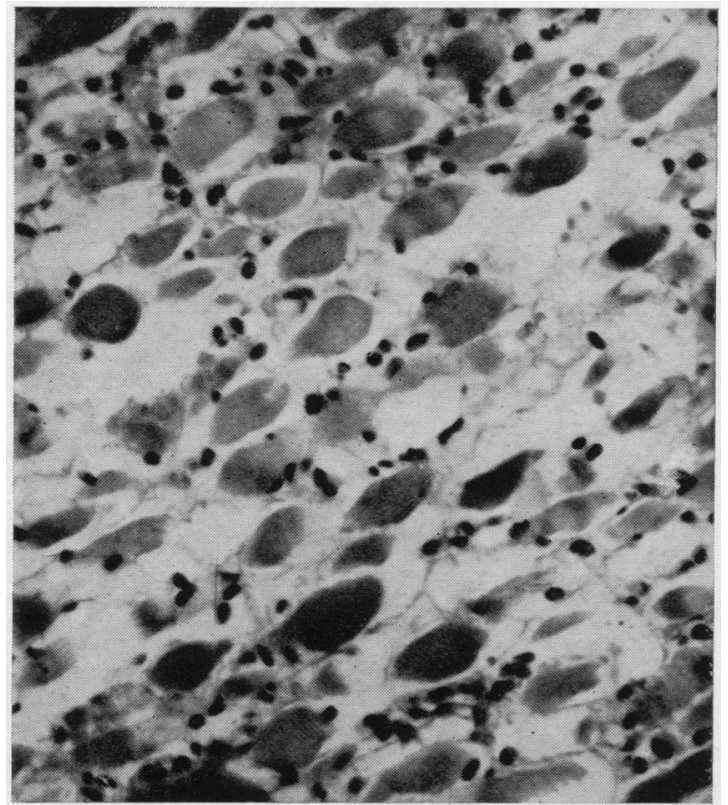

FIG. 5.-Case 2: microscopic appearance of the diaphragm $(\times 250)$.

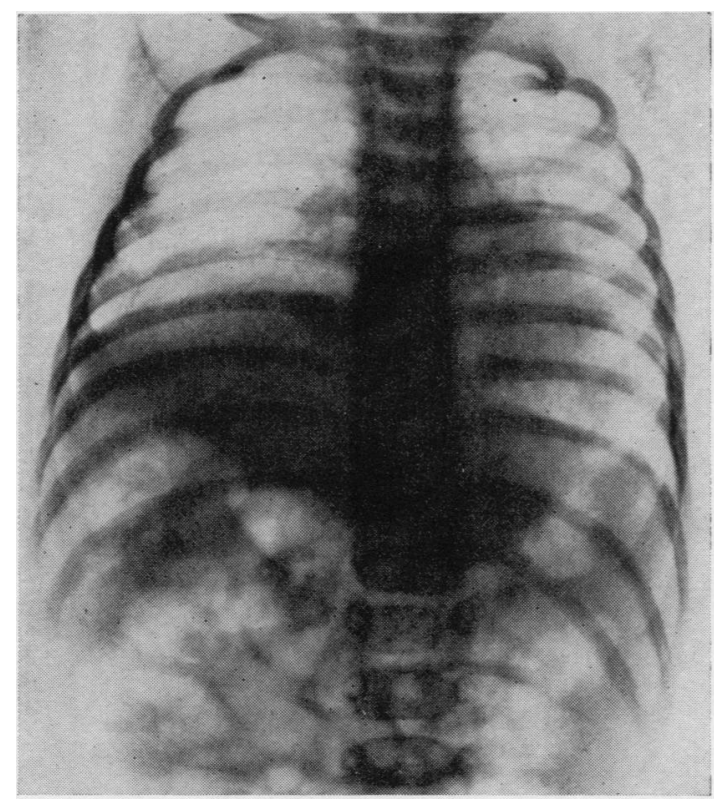

FIG. 6.-Case 2: radiograph showing improvement a few days after operation.

been no trouble of any sort. On screening, the right side of the diaphragm still showed paradoxical movement on respiration, but seven months after operation the right diaphragm, though still slightly elevated, was moving synchronously on inspiration.

\section{Discussion}

NATURE OF THE LESION.-When associated with an Erb's palsy the paralysed diaphragm is clearly part of a brachial plexus birth injury. Though it is somewhat difficult to see how birth trauma can cause an injury to the phrenic nerve and spare the rest of the brachial plexus, this seems to be the most likely cause even when the diaphragmatic paralysis is an isolated lesion. Several of the recorded cases have, like the two cases reported here, been breech presentations. In Case 2 biopsy of the diaphragm showed muscle degenerative changes typical of nerve injury, and in a case reported by Blattner (1942), which ended fatally at six months, section of the diaphragm also showed degenerative changes, and in sections of the phrenic nerve very few nerve axons could be seen. As with injuries to other nerves, the resulting paralysis may be transient, of considerable duration, or permanent. There are two cases reported of infants with diaphragmatic paralysis diagnosed within a few days of birth in whom the diaphragm recovered its function within a fortnight (Tyson and Bowman, 1933 ; Greenebaum and Harper, 1946). In others recovery has taken place after several months, and in yet others the paralysis was apparently permanent.

The Place of Surgical Treatment.-It is clear that not all cases require surgical treatment. But, as already mentioned, some cases terminate fatally. Of 13 reported cases which I have studied, some associated with brachial paralysis and some isolated diaphragmatic paralysis, five were fatal, death occurring at ages ranging from 24 hours to 6 months. Because of the striking and immediate relief of all respiratory difficulty achieved by operation in the two cases reported in this paper, I submit that operation should be undertaken if an infant should appear in any danger of dying because of this condition.

The principle I have used is to tighten the diaphragm so as to correct the high position of the dome and allow the mediastinum to return towards its normal position. This could be achieved by a series of plicating sutures, by producing one or more folds or pleats across the diaphragm, or, as in the present cases, by making an incision across the diaphragm a few centimetres from its costal attachment, perhaps combined, as in Case 2, with excision of a part of the diaphragm, and overlapping the two portions.

Relationship to So-Called Eventration of THE Diaphragm.-I stated earlier that no previously reported cases of diaphragmatic paralysis 
in the newborn have been operated on. But it should be added that operations such as those described above have been carried out for some cases of so-called " eventration" of the diaphragm, both in childhood and in adult life, and it is more than likely that many of these cases are, in reality, examples of diaphragmatic paralysis dating from infancy. When a thin, attenuated, high diaphragm is found in later life this could be the result of a longstanding paralysis. And when a high diaphragm is found on screening to show slight synchronous movement on respiration and not paradoxical movement, this could be due to incomplete recovery of a paralysed diaphragm. Even when only part of the diaphragm is thinned and elevated, this may be explained by normal innervation having returned to one part of the diaphragm and not to another.

An objection to this view is that most cases of eventration discovered later in life are on the left side, whereas, as stated earlier, most of the reported cases of diaphragmatic paralysis in the newborn have been right-sided. It is possible that a solid organ like the liver, when displaced upwards beneath a paralysed diaphragm in infancy, produces a greater degree of mediastinal shift and thus more severe symptoms than does a hollow viscus such as the stomach. In this way a right-sided paralysis would have attention drawn to it in the neonatal period by respiratory distress or, probably more often, would early prove fatal without being recognized, while a left-sided paralysis might not cause much trouble in infancy and be discovered later, either accidentally or because of pain and dyspepsia. Paralysis of the diaphragm due to birth trauma may be equally common on the two sides.

Whether an elevated diaphragm should ever be known as an eventration of the diaphragm is open $\cong$ to dispute, for the word "eventration," strictly speaking, means protrusion from the abdominal cavity, and should be used only with reference to abdominal viscera and not with reference to the diaphragm.

\section{SUMMARY}

Two cases of isolated unilateral paralysis of the $N$ diaphragm in newborn infants are described. These $\vec{\infty}$ are the twelfth and thirteenth examples of the con- 은 dition to be reported.

Both infants were operated on with very gratify- $\mathbb{C}$ ing results, and it is recommended that operation $\frac{}{\mathbb{T}}$ should be undertaken if life should be endangered.

The possible relationship of this condition to $\mathbb{D}$ cases of so-called "eventration" of the diaphragm is discussed.

It is a pleasure to express my appreciation of the skill and care shown in looking after these infants by the nursing staff and the resident medical staff of the hospital. I wish to thank Dr. Douglas Boyd for the radiographs and the facilities of his department, and $\stackrel{0}{\circ}$ Mr. David Mehaffey, A.R.P.S., for the photomicro- $\mathbb{Q}$ graph.

\section{REFERENCES}

Blattner, R. J. (1942). J. Pediut., 20, 223.

Greenebaum, J. V., and Harper, F. G. (1946). Ibid., 28, 483. Schifrin, N. (1952). Pediatrics, 9, 69.

Ty son, R. M., and Bowman. J. E. (1933). Amer. J. Dis. Child., 46, 30. 